University of Nebraska - Lincoln

DigitalCommons@University of Nebraska - Lincoln

P. F. (Paul Frazer) Williams Publications

Electrical \& Computer Engineering, Department

April 2003

\title{
The effect of plasma shielding on dust particle interaction in low pressure plasmas
}

\author{
M. E. Marks \\ University of Nebraska-Kearney, Kearney, Nebraska \\ P. F. Williams \\ University of Nebraska - Lincoln, pfw@moi.unl.edu
}

Follow this and additional works at: https://digitalcommons.unl.edu/elecengwilliams

Part of the Electrical and Computer Engineering Commons

Marks, M. E. and Williams, P. F., "The effect of plasma shielding on dust particle interaction in low pressure plasmas" (2003). P. F. (Paul Frazer) Williams Publications. 25.

https://digitalcommons.unl.edu/elecengwilliams/25

This Article is brought to you for free and open access by the Electrical \& Computer Engineering, Department of at DigitalCommons@University of Nebraska - Lincoln. It has been accepted for inclusion in P. F. (Paul Frazer) Williams Publications by an authorized administrator of DigitalCommons@University of Nebraska - Lincoln. 


\title{
The effect of plasma shielding on dust particle interaction in low pressure plasmas
}

\author{
M. E. Markes ${ }^{\text {a) }}$ \\ Department of Physics, University of Nebraska-Kearney, Kearney, Nebraska 68849 \\ P. F. Williams \\ Department of Electrical Engineering, University of Nebraska-Lincoln, Lincoln, Nebraska 68588
}

(Received 12 September 2002; accepted 17 January 2003)

\begin{abstract}
The interaction of charged dust particles in plasma is often modeled assuming a constant charge on each particle with the particle charge determined by requiring zero net current to the particles when they are far apart. In this paper the variation in particle charge is calculated for two-body encounters of dust particles taking into consideration mutual electrostatic shielding and a finite Debye length $\lambda_{D}$. Results are reported for particle radii between $0.0105 \lambda_{D}$ and $0.349 \lambda_{D}$. Work is also calculated as a function of particle separation, and it is shown that dust particle energies greater than $50 \mathrm{eV}$ are required before the variation in charge becomes significant. It is also demonstrated that for particles with radii greater than $\lambda_{D} / 10$ the plasma can act to increase the force at separations less than $3 \lambda_{D}$, and it is shown that the Debye-Hückel factor $\left(1+a / \lambda_{D}\right)$ may overestimate particle charge by as much as ten percent for larger particles. (C) 2003 American Institute of Physics.
\end{abstract}

[DOI: $10.1063 / 1.1559682$ ]

\section{INTRODUCTION}

In an rf-driven plasma the greater speed of the electrons relative to the ions produces a charging mechanism for dust particles introduced into the plasma. ${ }^{1,2}$ The relatively greater flux of electrons charges the dust particle surface increasingly more negative until the enhanced ion flux to the surface balances the electron flux. In many theoretical studies of dusty-plasma phase transitions, dust transport, two-body interactions, and dust-particle agglomeration, the charge on individual dust particles has been assumed constant during particle-particle interactions. ${ }^{3-6}$ However, the charge on dust particles in a plasma can vary for many reasons. ${ }^{7-10}$ One cause is the spatial variation in the electron velocity distribution function. This variation will produce a larger electron flux to the particle surface in regions with larger average electron speed, and a more negative potential on the particle surface is required in these regions to balance the flux of ions and electrons. A second cause is the presence of other dust particles that can reduce the density of electrons available to charge a given particle. This effect may be present even for pairs of dust particles. ${ }^{10}$ A third mechanism is mutual electrostatic shielding within a group of two or more dust particles. In systems with mutual electrostatic shielding the particles are sufficiently close that their individual fields combine so that the steady-state floating potential is obtained with less charge per particle.

A special case of mutual electrostatic shielding occurs when two otherwise isolated particles interact. Typical ion/ electron currents to the surface of a dust particle exceed $10^{7}$ per second, and a small $(1 \mu \mathrm{m})$ dust particle may have an excess charge $\sim 10^{3}$ electrons and a speed of only a few

${ }^{a)}$ Electronic mail: markesme@unk.edu centimeters per second. With an ion/electron current of this magnitude the dust particle charge varies quasi-statically with particle separation and the question of how much charge variation may be expected during a two-body encounter arises.

A previous study by Choi and Kushner (CK) using particle-in-cell monte-carlo methods has indicated that a variation of particle charge occurs during two-body encounters of co-axial cylindrical dust particles in plasma. ${ }^{10}$ In addition to being based on a cylindrical particle geometry and Monte carlo methods, this study included the assumption that ions incident on a particle surface are absorbed with unit probability. In this paper we present a deterministic solution of a similar problem with the focus of attention on the mutual electrostatic interaction between the particles. Implicit in our approach are the assumptions that the dust particles can be modeled as ideal conductors and the ions and electrons are absorbed with relatively low probability by the dust particles. The assumption concerning ideal conducting spheres allows us to use the exact solution of two conducting spheres in vacuum as a reference system. The assumption concerning absorption probability allows us to use orbital motion limited (OML) theory. ${ }^{11-15}$ All relevant parameters such as the floating potential and particle charge are calculated using OML theory. The force and charge associated with equivalent particles in a vacuum are obtained from the method of images. ${ }^{16}$ Our results generally support and to some degree complement the results of CK. However, the electron temperature assumed by $\mathrm{CK}(3.9 \mathrm{eV})$ was significantly higher than ours $(0.86 \mathrm{eV})$.

In Sec. II we use OML theory to develop a model for plasma space-charge density as a function of potential and calculate the electron and ion currents to the dust particles and the floating potential. OML theory is based on the as- 


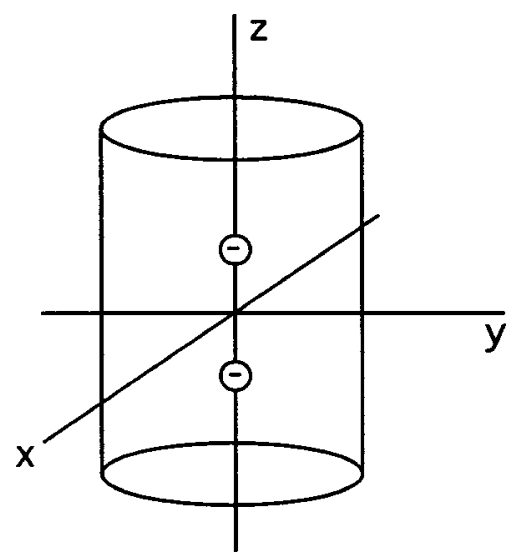

FIG. 1. Problem space for two-particle system.

sumption of a collisionless sheath introduced by Langmuir and Mott-Smith to model electrical probes in plasmas. ${ }^{17-19}$ In Sec. II we also discuss the validity of OML theory with respect to the two-particle system. In Sec. III the chargedensity function is substituted into Poisson's equation, and the method of finite elements and successive over-relaxation is used to obtain the potential for the two-particle nonlinear system. In Sec. IV we calculate the interparticle force, dustparticle charge, and work to bring the particles together as functions of particle separation. We also discuss the relationship between the interparticle force and the Helmholtz free energy of the ion and electron Maxwellian gases. Three particle radii are considered: $0.0105 \lambda_{D}, 0.140 \lambda_{D}$, and $0.349 \lambda_{D}$. In Sec. V we present our conclusions.

\section{SPACE-CHARGE DENSITY AND FLOATING POTENTIAL}

We begin by considering an unmagnetized plasma in which the electrons and ions can be regarded as classical particles and in which electron-neutral and ion-neutral collisions can be neglected within a finite region of space that contains the two spherical dust particles that are assumed to be ideal electrical conductors. We shall take this volume to be a cylinder as shown in Fig. 1. Within the volume, in steady-state, the electron and ion distribution functions $f$ are solutions to Vlasov equations of the form

$$
-\frac{\partial f}{\partial t}=\boldsymbol{v} \cdot \nabla_{r} f+\frac{q}{m} \boldsymbol{E} \cdot \boldsymbol{\nabla}_{v} f=0 .
$$

Here $q$ is the absolute value of the electronic charge, $m$ is the mass of the ion or electron, and $\boldsymbol{E}$ is the self-consistent electric field. For simplicity we consider only ions with a single positive charge, and we assume that all positive-energy ion and electron states are found on orbits that map back to the boundary of the collisionless region: i.e., we apply the OML method.

The main caveats of the OML method are: (1) A steadystate, (2) collisionless ions and electrons, and (3) the orbit of any ion or electron can be traced back to the boundary of the collisionless region where the potential vanishes. Lampe ${ }^{15}$ has recently shown that the OML theory for isolated particles is a good approximation if

$$
0.03 \frac{a}{\lambda_{D}} \frac{T_{e}}{T_{i}} \ll 1
$$

where $a$ is the radius of the particle, $\lambda_{D}$ is the Debye length, $T_{i}$ is the ion temperature, and $T_{e}$ is the electron temperature. In this paper we report results for particles such that

$$
0.03 \frac{a}{\lambda_{D}} \frac{T_{e}}{T_{i}}<0.21
$$

It is caveat (3) that fails to be satisfied for a system of two dust particles that are opaque to the plasma. However, before we discuss the limitations of the OML method as applied to a two-particle system, we shall derive plasma space-charge density as a function of potential by assuming both particles are to a good approximation transparent to ions and electrons, and we shall derive the floating potential of particle 1 by assuming particle 2 takes on the true (but unknown) floating potential of the two-particle system but remains transparent to ions and electrons. After these quantities have been obtained, we shall discuss the probable impact of these approximations.

We begin by observing that Eq. (1) is essentially a statement of Liouville's theorem which implies the distribution function $f$ is constant along all phase-space trajectories in the collisionless region. Given the capture probability for ions and electrons within the dust particles is small, most internal phase-space points with positive energy map into points on the boundary of the collisionless region for both ions and electrons, and the negative-energy states for ions are unoccupied if collisions with neutrals are neglected. If the distribution functions $f$ for the ions and electrons are Maxwellian on the boundary (where the potential vanishes), then the distribution functions $f$ are

$$
f(\boldsymbol{r}, \boldsymbol{v})=N_{0}\left(\frac{m}{2 \pi k_{B} T}\right)^{3 / 2} e^{-m / 2 k_{B} T\left(v^{2}+2 \beta \psi\right)} .
$$

Here $N_{0}$ is the bulk plasma density, $\beta$ is the charge-to-mass ratio for the ion or electron and $\psi$ is the electrostatic potential.

The number densities for electrons and ions can be obtained by integrating the distribution functions over velocity space. ${ }^{11,14}$ The result is that the number density for electrons is

$$
n_{e}=N_{0} e^{q \psi / k_{B} T_{e}},
$$

where $k_{B}$ is Boltzmann's constant and $\beta_{e}$ has been replaced by $-q / m_{e}$, and the result for ions is

$$
n_{i}=N_{0}\left[2 \sqrt{\frac{-q \psi}{\pi k_{B} T_{i}}}+e^{-q \psi / k_{B} T_{i}} \operatorname{erfc}\left(\sqrt{\frac{-q \psi}{k_{B} T_{i}}}\right)\right],
$$

where erfc is the complementary error function. Here it should be noted that for ions the electrostatic force is attractive, and the lower limit on the ion speed is $\sqrt{-2 \beta_{i} \psi}$. Combining Eqs. (3) and (4), the net space-charge density for transparent dust particles is 


$$
\begin{aligned}
\rho_{s c}= & q N_{0}\left\{2 \sqrt{\frac{-q \psi}{\pi k_{B} T_{i}}}+e^{-q \psi / k_{B} T_{i}}\right. \\
& \left.\times \operatorname{erfc}\left(\sqrt{-\frac{q \psi}{k_{B} T_{i}}}\right)-e^{q \psi / k_{B} T_{e}}\right\} .
\end{aligned}
$$

In order to obtain an estimate of the floating potential in the two-particle system, we now consider particle 1 to be opaque. However, particle 2 is assumed to remain transparent and to be held at the true (but unknown) floating potential of the two-particle system. The flux $\Phi$ impinging on the surface of particle 1 is

$$
\Phi=-\int \boldsymbol{v} \cdot \hat{a} f_{a}\left(\boldsymbol{v}, \boldsymbol{r}_{a}\right) d^{3} v,
$$

where $\boldsymbol{r}_{a}$ is a point on the particle's surface, $\hat{a}$ is an outward unit vector normal to the surface, and $f_{a}$ is the distribution function for ions or electrons at the surface of particle 1 . Assuming a spherical particle with a uniform hemispherical velocity distribution for the electrons at the surface as given by Eq. (2), the resulting electron particle current to the surface of particle 1 is

$$
I_{e}=4 \pi a^{2} \Phi_{e}=\pi a^{2} N_{0}\left(\frac{8 k_{B} T_{e}}{\pi m_{e}}\right)^{1 / 2} e^{q \psi_{a} / k_{B} T_{e}} .
$$

Here $\psi_{a}$ is the surface potential of the particle, $a$ is the particle radius, and $I_{e}$ is the electron particle current to the surface of particle 1 .

The ion flux to the surface of particle 1 is obtained in a similar way except that a lower limit on the speed $\sqrt{-2 \beta_{i} \psi_{a}}$ is used due to the attractive nature of the potential for ions. The resulting ion current to the surface is

$$
I_{i}=4 \pi a^{2} \Phi_{i}=\pi a^{2} N_{0}\left(\frac{8 k_{B} T_{i}}{\pi m_{i}}\right)^{1 / 2}\left[1-\frac{q \psi_{a}}{k_{B} T_{i}}\right] .
$$

Demanding $I_{e}=I_{i}$ yields

$$
e^{q \psi_{a} / k_{B} T_{e}}=\left(\frac{m_{e} T_{i}}{m_{i} T_{e}}\right)^{1 / 2}\left[1-\frac{q \psi_{a}}{k_{B} T_{i}}\right] .
$$

This transcendental equation can be solved to obtain the floating potential on the surface of particle 1 when particle 2 is transparent and held at the true floating potential of the two-dust-particle system. This potential is identical to the potential of an isolated dust particle. For an argon plasma with $N_{0}=10^{15} \mathrm{~m}^{-3}, T_{e}=10^{4} \mathrm{~K}$, and $T_{i}=500 \mathrm{~K}$ the result is $\psi_{a}=-2.58 \mathrm{~V}$.

We will now discuss the change in the floating potential on particle 1 as particle 2 becomes opaque. Since the OML method does not depend on the symmetry of the potential, particle 1 will take on the same floating potential as that of an isolated particle as long as particle 2 remains transparent. Thus, within the context of the OML theory, the only difference between the floating potential on an isolated particle and the floating potential on a dust particle in a system of two opaque dust particles must be due only to the relative change between ion and electron currents incident on particle 1 when particle 2 becomes opaque. An estimate of the effect of this change can be obtained from the results of Choi and

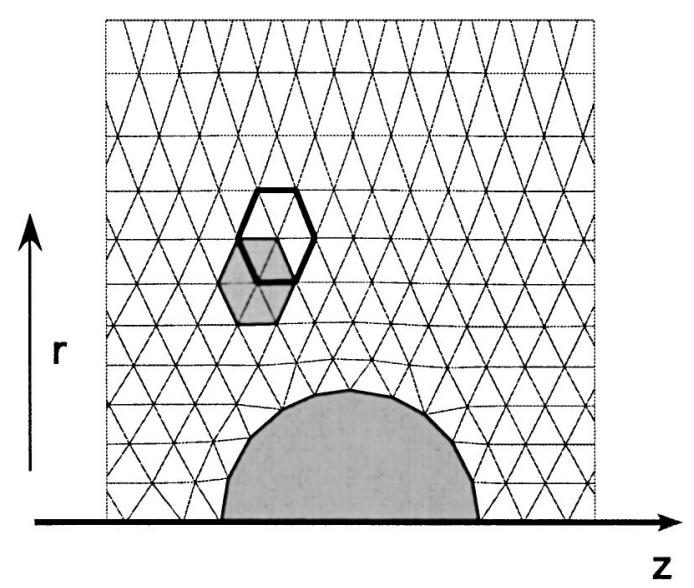

FIG. 2. The computational mesh near a dust particle.

Kushner. ${ }^{10}$ They found that for two co-axial cylindrical dust particles, the floating potential varied by about 14 percent as the particles were brought together. A variation of this size would not significantly affect the main conclusions of this study. Therefore, we shall adopt the floating potential of an isolated dust particle $(-2.58 \mathrm{~V})$ as the floating potential for our two particle system.

With respect to the space-charge density calculated on the assumption of transparent particles, it may be observed that this assumption mainly introduces an error in space charge density near the particle surfaces, and this error is at most given by a factor of two. When the particles are relatively close together, the interparticle force is dominated by the charge on the particles. This is because there are many more electrons bound to the dust particles than there are excess ions in the region around the particles. Since all quantities reported in this study either depend on force or particle charge, only a small error will result from the error in space charge density near the particles.

\section{THE TWO-PARTICLE POTENTIAL}

With the space-charge density given by Eq. (5), Poisson's equation becomes

$$
\begin{aligned}
\frac{1}{r} \frac{\partial}{\partial r}\left[r \frac{\partial \psi}{\partial r}\right]+\frac{\partial^{2} \psi}{\partial z^{2}} & \\
= & -\frac{q N_{0}}{\varepsilon_{0}}\left\{2 \sqrt{\frac{-q \psi}{\pi k_{B} T_{i}}}+e^{-q \psi / k_{B} T_{i}}\right. \\
& \left.\times \operatorname{erfc}\left(\sqrt{-\frac{q \psi}{k_{B} T_{i}}}\right)-e^{q \psi / k_{B} T_{e}}\right\} .
\end{aligned}
$$

Here $\varepsilon_{0}$ is the electric permittivity of free space. The physical boundary conditions are that the potential goes to zero at infinity and takes on the value given by Eq. (9) on the surface of the particles. Inside the problem cylinder Eq. (10) can be solved numerically using the method of finite elements and successive over-relaxation. ${ }^{20}$

In the method of finite-elements a variable-resolution triangular mesh is used. The triangles defined by the mesh can be grouped into overlapping hexagonal regions of six tri- 
angles each as shown in Fig. 2. A potential is calculated or assigned for each vertex of the mesh. The relationship between the potentials of a hexagonal element is ${ }^{20}$

$$
\psi_{0} \sum_{n=1}^{6} W_{n}-\sum_{n=1}^{6} W_{n} \psi_{n}=\frac{1}{\varepsilon_{0}} \sum_{m=1}^{6} \frac{\bar{r}_{m} \rho_{m} \tau_{m}}{3} .
$$

Here $\psi_{0}$ is the potential of the central vertex, $n$ is a local index for the six perimeter vertices, and $m$ is a local index for the six triangular regions of the hexagonal element. The six triangular regions have areas $\tau_{m}$ and charge densities $\rho_{m}$, and the parameters $\bar{r}_{m}$ are the radial coordinates of the centroids of the triangular regions. The charge density within a triangular region $m$ is taken to be the average of the charge densities calculated at the vertices of the triangle using Eq. (5). The parameters $W_{n}$ are coupling constants which depend on the relative coordinates of the vertices of the hexagon:

$$
\begin{aligned}
W_{n}= & \bar{r}_{n+1}\left[\frac{Y_{n+1}\left(Y_{n+1}-Y_{n}\right)+X_{n+1}\left(X_{n+1}-X_{n}\right)}{4 \tau_{n+1}}\right] \\
& +\bar{r}_{n}\left[\frac{Y_{n-1}\left(Y_{n}-Y_{n-1}\right)+X_{n-1}\left(X_{n}-X_{n-1}\right)}{4 \tau_{n}}\right],
\end{aligned}
$$

where

$$
X_{n}=z_{n}-z_{0}, \quad Y_{n}=r_{n}-r_{0} .
$$

Equation (11) is applied to each vertex in the mesh with an unknown potential leading to a large set of coupled equations that can be solved to obtain the potential at each vertex.

Successive over-relaxation was performed using an alternating scheme to advance the potentials on odd-even vertices on odd-even values of the iteration index. The vertex potentials were iterated as specified by Eqs. (14) and (15)

$$
\begin{aligned}
& \psi_{0}^{(t+1)}=\psi_{0}^{(t)}+\omega \Delta \psi_{0}^{(t \rightarrow t+1)}, \\
& \Delta \psi_{0}^{(t \rightarrow t+1)}=\frac{\frac{1}{\varepsilon_{0}} \sum_{m=1}^{6} \frac{\bar{r}_{m} \rho_{m}^{(t)} \tau_{m}}{3}+\sum_{n=1}^{6} W_{n} \psi_{n}^{(t)}}{\sum_{n=1}^{6} W_{n}}-\psi_{0}^{(t)},
\end{aligned}
$$

where $t$ is the iteration index. The parameter $\omega$ is an overrelaxation parameter $1 \leqslant \omega<2$. In general, $\omega$ is chosen as large as convergence will allow. In practice the optimum $\omega$ is dependent on the particular computational mesh and the charge density function. For our problem a typical value of $\omega$ was about 1.2.

Figure 3 shows a plot of the calculated potential for a pair of $1 \mu \mathrm{m}$ diameter particles in plasma using the finiteelement method. The potential is plotted in an $r z$ plane with only a small part of the total computational volume shown. The plasma parameters are plasma density $N_{0}=10^{15} \mathrm{~m}^{-3}$, electron temperature $T_{e}=10^{4} \mathrm{~K}$, and ion temperature $T_{i}$ $=500 \mathrm{~K}$. The center-to-center distance is one Debye unit $\lambda_{D}$

$$
\lambda_{D}=\left\{\frac{q^{2} N_{0}}{k_{B} \varepsilon_{0}}\left(\frac{1}{T_{i}}+\frac{1}{T_{e}}\right)\right\}^{-1 / 2} .
$$

For these plasma parameters $\lambda_{D}=47.6 \mu \mathrm{m}$.

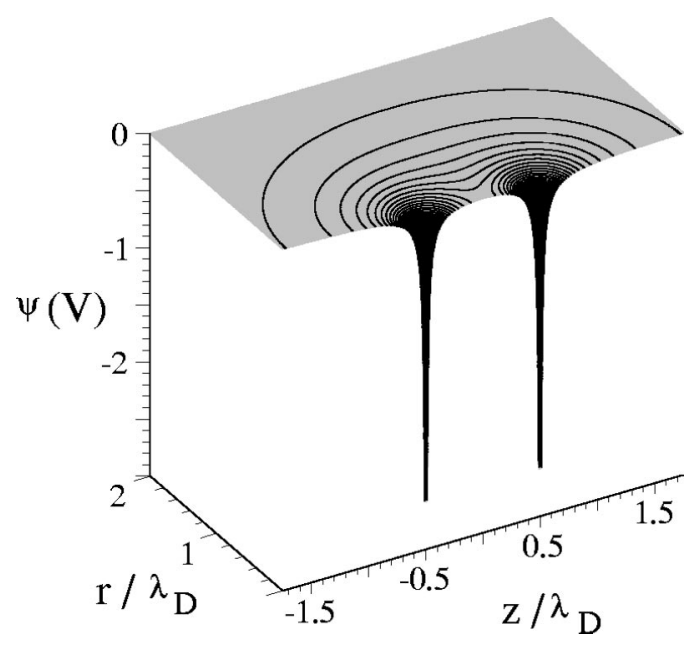

FIG. 3. Sample finite-element solution.

\section{FORCE AND PARTICLE CHARGE}

The force was obtained using an expression for the force between two thin coaxial charged rings obtained from Smythe ${ }^{21}$

$$
\begin{aligned}
F_{i, j}= & \frac{2 \bar{r}_{i} \bar{r}_{j}\left(\bar{z}_{j}-\bar{z}_{i}\right) \lambda_{i} \lambda_{j}}{\varepsilon_{0}\left[\left(\bar{r}_{j}-\bar{r}_{i}\right)^{2}+\left(\bar{z}_{j}-\bar{z}_{i}\right)^{2}\right]} \\
& \times \frac{E\left(\sqrt{\frac{4 \bar{r}_{i} \bar{r}_{j}}{\left(\bar{r}_{j}+\bar{r}_{i}\right)^{2}+\left(\bar{z}_{j}-\bar{z}_{i}\right)^{2}}}\right)}{\sqrt{\left(\bar{r}_{j}+\bar{r}_{i}\right)^{2}+\left(\bar{z}_{j}-\bar{z}_{i}\right)^{2}}} .
\end{aligned}
$$

Here $\left(\bar{z}_{i}, \bar{r}_{i}\right)$ and $\left(\bar{z}_{j}, \bar{r}_{j}\right)$ are the axial positions and radii of the two rings, $\lambda_{i}$ and $\lambda_{j}$ are the linear charge densities, and $E$ is a complete elliptic integral of the second kind. ${ }^{22}$

It might be asked why the force is not calculated from the electrostatic potential energy, since charge density and electrostatic potential are available from the solution of the Poisson problem. The answer is that the sheath-particle system is not closed. It exchanges ions and energy with the Maxwellian ion environment, and it exchanges electrons and energy with the Maxwellian electron environment. Since the volumes and temperatures of the overall ion and electron systems may be considered fixed, it is the Helmholtz free energy $F_{H}$ that determines the interparticle force $F$

$$
F=-\frac{\partial F_{H}}{\partial r} .
$$

Here $r$ is the interparticle separation, and the ion and electron temperatures are held constant. Although we have presently not completed a Helmholtz free energy calculation for the system under consideration, we have done so in the Debye approximation for dust particles in an ion-neutral plasma in a uniform background of negative charge density. For this system it can be shown that the interparticle force predicted on the basis of the Helmholtz free energy is identical to that calculated from a direct application of Coulomb's law. Of course, since the interparticle force must ultimately have a physical origin, this result is to be expected. However, we are 
currently studying the possible application of statistical thermodynamics to investigate the trapped-ion issue discussed in Sec. II.

Equation (17) was applied to the dust particles by assuming the surface of each particle was represented by ten rings $i$, one ring for each of the ten surface segments. The axial position and radius of a surface ring were determined by the mid-point of the line segment, and the surface-charge density was obtained from the normal component of the electric field calculated to second order from the potentials at the vertices on and near the segment. The linear charge density for a surface ring was obtained by multiplying the surface charge density by the segment length. However, an examination of the potential near the surface of an isolated spherical particle, obtained using the charge density given by Eq. (5) and a fourth-order Runge-Kutta algorithm, indicated that the mesh size used in the finite-element solution would underestimate the surface gradient by about 10 percent. This expectation was supported by two observations: First, the surface charge as calculated from the Runge-Kutta solution was about 12 percent more than the surface charge calculated for an identical, isolated particle using the finite-element method and the second-order estimate of the surface gradient using the computational mesh. The second supporting observation was that the total space charge obtained from the finiteelement solution was also 12 percent more than the surface charge as calculated using the second-order method. Therefore, before the force was calculated, the surface charge was scaled by a uniform factor to balance it with the total space charge. Thus, the system as a whole was forced to neutrality before each force calculation was performed, and the particle charge we report is the scaled particle charge.

The rings $j$ were associated with the space charge. Each triangular element of the mesh was represented by a ring, and each ring had a mirror-image ring in the $z<0$ region. The linear charge density for a space-charge ring was taken as the product $\tau_{m} \rho_{m}$. The axial position and radius of the ring were taken as the geometrical center (centroid) of the triangular element. The net force exerted on the dust particle in the computational mesh was obtained by a summation over ring pairs as

$$
F=\sum_{i} \sum_{j} F_{i, j}+\sum_{i} \sum_{i^{\prime}} F_{i, i^{\prime}} .
$$

Here the index $i$ is summed over the ten rings representing the particle surface, the index $j$ is summed over the rings representing the triangular plasma-volume elements and their images, and the index $i^{\prime}$ is summed over the ten rings of the mirror image dust particle.

In vacuum the force between identical conducting spheres of radius $a$ separated by a distance $r$ and held at a potential $\psi_{a}$ is obtained from the method of images ${ }^{16}$

$$
\begin{aligned}
& F=\left(\frac{d C_{11}}{d r}+\frac{d C_{12}}{d r}\right) \psi_{a}^{2}, \\
& C_{11}=4 \pi \varepsilon_{0} a \sinh (\beta(x)) \sum_{n=1}^{\infty} \operatorname{csch}[(2 n-1) \beta(x)],
\end{aligned}
$$
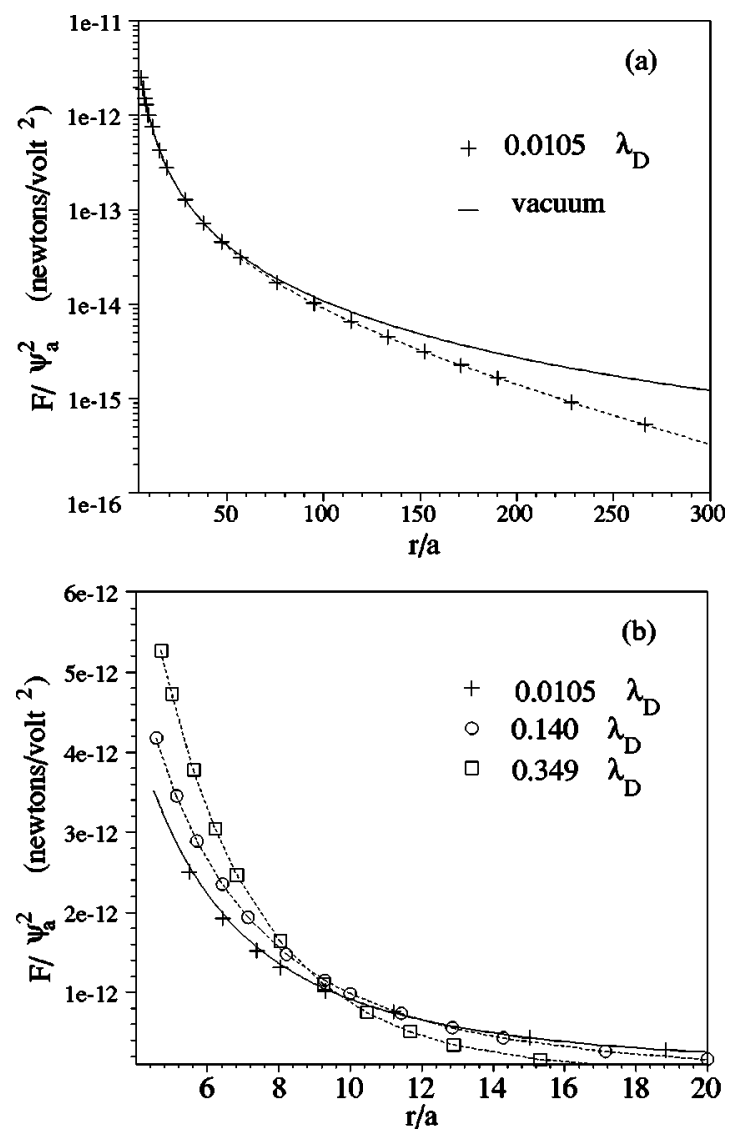

FIG. 4. Force as a function of particle separation.

$$
C_{12}=-4 \pi \varepsilon_{0} a \sinh (\beta(x)) \sum_{n=1}^{\infty} \operatorname{csch}[2 n \beta(x)],
$$

and

$$
\beta(x)=\cosh ^{-1}(x / 2) .
$$

Here $x=r / a$, where $r$ is the center-to-center separation and $a$ is the radius. $C_{11}$ and $C_{12}$ are the self and mutual capacitances of the particles. The charge on one of the particles is given by

$$
Q=\left(C_{11}+C_{12}\right) \psi_{a} .
$$

Figure 4(a) shows a plot of the net interparticle force for small particles with radii $0.0105 \lambda_{D}$. All particles (even those in vacuum) are assumed to be held at the floating potential of the plasma, and the axes are scaled as suggested by Eqs. (20)-(23). With this choice of scaling the interparticle force per square volt in vacuum falls on the same curve for all particle sizes. This curve is shown as the solid line in Fig. 4(a). It can be seen that for particles with radii much less than $\lambda_{D}$ we obtain the expected result that, when the particle separation is much less than $\lambda_{D}$, the interparticle force is essentially the same as that calculated for equivalent particles in vacuum, and that when $r$ approaches $\lambda_{D}($ at $r / a \approx 95)$ the interparticle force begins to show shielding by the plasma.

Figure 4(b) shows a similar plot, on a linear force per square volt scale, with the other two particle sizes also represented. Again all particles are held at the floating potential 


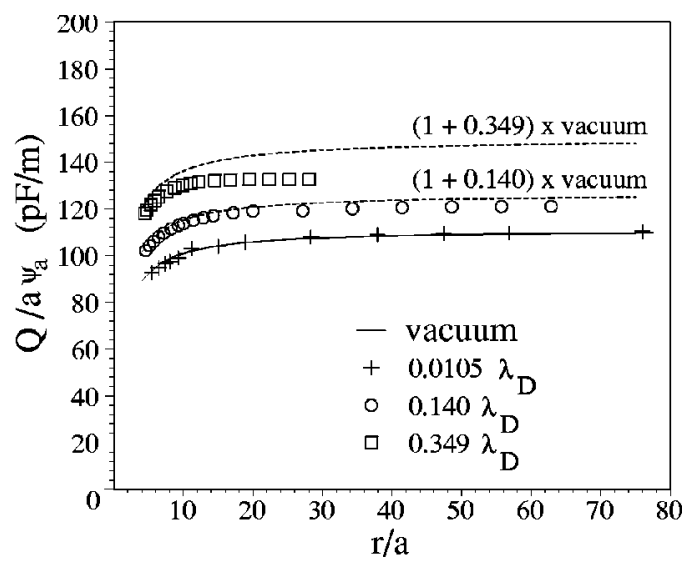

FIG. 5. Total capacitance per unit radius.

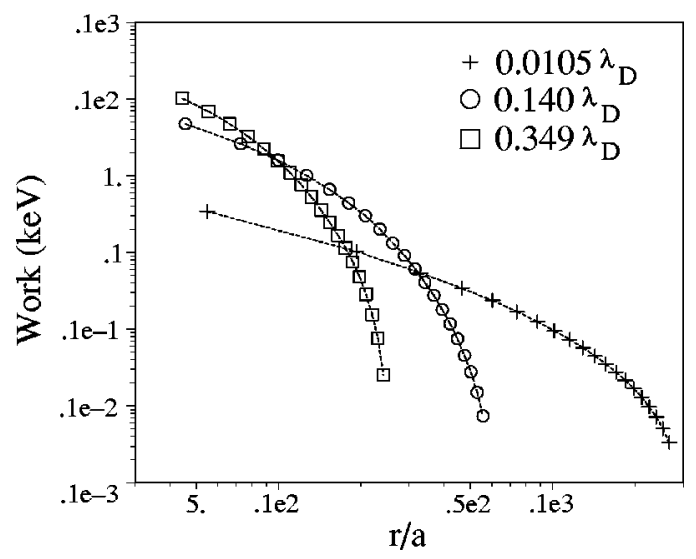

FIG. 6. Work as a function of particle separation. of the plasma, and in vacuum spherical particles of all sizes fall on the same curve which is shown as a solid line. Over this range of $r / a$ the small particles are well inside the Debye shielding distance and fall almost exactly on the vacuum curve. However, the larger particles show two types of behavior relative to the vacuum. At larger values of $r / a$ they show shielding by the plasma, but at smaller separations they show an enhanced force relative to the vacuum. The enhanced force relative to the vacuum is due to an increased capacitance and charge for particles in plasma. Both the mutual and self-capacitances are increased relative to the vacuum. It can be seen that for particles with radii less than about $1 / 10 \lambda_{D}$ the effect is negligible. However, for larger particles the effect is significant.

For isolated particles it is a common procedure to calculate dust-particle charge using the free-space capacitance $C$ multiplied by a factor based on the Debye-Hückel approximation for the potential

$$
C=4 \pi \varepsilon_{0} a\left(1+a / \lambda_{D}\right) .
$$

Thus, for a floating potential $\psi_{a}$ the charge on an isolated dust particle is

$$
Q=4 \pi \varepsilon_{0} a \psi_{a}\left(1+a / \lambda_{D}\right) .
$$

Given the Debye potential, the charge $Q$ is exact. It can be seen from Eq. (26) that the charge on an isolated dust particle begins to increase as $a \rightarrow \lambda_{D}$.

It has been observed that the Debye approximation for the potential becomes increasingly inaccurate as the ratio of particle radius to Debye length increases. ${ }^{14}$ It is of some practical interest to determine the range of particle sizes over which Eq. (26) can be applied. Figure 5 shows the total capacitance per unit particle radius: i.e., the particle charge divided by the product of floating potential and particle radius as a function of $r / a$. With this choice of scaling all particle pairs in vacuum are represented by the same curve which is shown as a solid line. The dashed lines in this figure represent the vacuum curve multiplied by the Debye-Hückel factor $\left(1+a / \lambda_{D}\right)$ for the two larger particles. For the small particles $\left(1+a / \lambda_{D}\right)$ is to a good approximation unity, and it can be seen that the capacitance approximately equals the vacuum capacitance for all particle separations. For the
$0.140 \lambda_{D}$ particles the Debye-Hückel factor predicts a slightly higher charge for all particle separations but still gives a good approximation to the particle capacitance for all separations. However, for the $0.349 \lambda_{D}$ particles the factor is observed to overestimate the charge with the error for the well-separated particles being somewhat larger than for interacting particles.

It has been calculated that dust particles in laboratory plasmas can be accelerated to energies on the order of $4 \mathrm{keV}$ by transfer of momentum from ions and neutrals, electric fields, and other effects. ${ }^{10}$ Thus the question may be asked whether or not a variation in particle charge needs to be considered during dust-particle interactions in plasma. Figure 6 shows a plot of the work required by an external applied force to bring a dust particle from infinity to a specified separation $r / a$ relative to a second fixed particle. From Fig. 5 we see that the particle charge begins to vary for values of $r / a<20$, and Fig. 6 shows that energies on the order of 50 $\mathrm{eV}$ are required to bring the particles to this separation.

\section{CONCLUSIONS}

A comparison of our results with those of Choi and Kushner $^{10}$ (CK) reveals that the most significant difference between our findings and theirs is that they calculate a 14percent variation in floating potential as the particles are brought together. They find that, as the particles are brought together, the floating potential first decreases in magnitude by about 14 percent. As the particles are brought closer together, the floating potential increases in magnitude and again takes on the single-particle value. CK attribute this effect to the repulsion of electrons away from the first particle by the field due to the second particle. However, as discussed in Sec. II, for ideal conductors this effect must be due to a mutual shadowing of ion and electron currents to the particle surfaces. Since CK uniformly distributed the net collected charge over the surfaces of the particles, their results should vary slightly from that of ideal conductors. It is possible that the 14-percent variation in floating potential may be in part due to the low conductivity of the particles.

The results of our study indicate that the particle charge, during two-particle interactions in a plasma for dust particles with radii less than about $\lambda_{D} / 10$, can be calculated using a 
factor of $\left(1+a / \lambda_{D}\right)$ times the self and mutual vacuum capacitances for all particle separations. However, this procedure overestimates the charge on larger particles by as much as ten percent. This result is no doubt due at least in part to the failure of the Debye model for particles with radii approaching a Debye length. In addition, by making a direct comparison with vacuum results, we have found that the force and charge for dust particles in a plasma can exceed the force and charge for equivalent particles held at the same potential in a vacuum if the particles are in close proximity and have radii larger than about $\lambda_{D} / 10$. We have also found that the charge on interacting dust particles can be considered constant during two-body encounters if the dust-particle energies are less than about $50 \mathrm{eV}$. This means the variation in charge during two-body encounters may need to be considered in some but not all systems.

\section{ACKNOWLEDGMENTS}

In this work we have benefited greatly from conversations with Mark Kushner, John Goree, and Martin Lampe.

This work was supported by the State of Nebraska through a grant from the Nebraska Research Initiative.

${ }^{1}$ J. P. Boeuf and C. Punset, in Dusty Plasmas: Physics, Chemistry and Technological Impacts in Plasma Processing, edited by A. Bouchoule (John Wiley and Sons, New York, 1999), Chap. 1.
${ }^{2}$ P. K. Shukla, Phys. Plasmas 8, 1791 (2001).

${ }^{3}$ R. T. Farouki and S. Hamaguchi, Appl. Phys. Lett. 61, 2973 (1992).

${ }^{4}$ F. Melandso and J. Goree, J. Vac. Sci. Technol. A 14, 511 (1996).

${ }^{5}$ F. Y. Huang, H. H. Hwang, and M. J. Kushner, J. Vac. Sci. Technol. A 14, 562 (1996).

${ }^{6}$ D. P. Resendes, J. T. Mendonca, and P. K. Shukla, Phys. Lett. A 239, 181 (1998).

${ }^{7}$ C. K. Goertz and W. H. Ip, Geophys. Res. Lett. 11, 349 (1984).

${ }^{8}$ E. C. Whipple, T. G. Northrop, and D. A. Mendis, J. Geophys. Res. 90, 7405 (1985)

${ }^{9}$ C. K. Goertz, Rev. Geophys. 27, 271 (1989).

${ }^{10}$ S. J. Choi and M. J. Kushner, J. Appl. Phys. 75, 3351 (1994).

${ }^{11}$ J. G. Laframboise and L. W. Parker, Phys. Fluids 16, 629 (1973).

${ }^{12}$ J. E. Allen, Phys. Scr. 45, 497 (1992).

${ }^{13}$ J. E. Allen, B. M. Annaratone, and U. de Angelis, J. Plasma Phys. 63, 299 (2000).

${ }^{14}$ M. Lampe, G. Joyce, G. Ganguli, and V. Gavrishchaka, Phys. Plasmas 7, 3851 (2000)

${ }^{15}$ M. Lampe, J. Plasma Phys. 65, 171 (2001).

${ }^{16}$ W. R. Smythe, Static and Dynamic Electricity (McGraw-Hill, New York, 1968), Chap. 5, pp. 131-132, 3rd ed.

${ }^{17}$ H. M. Mott-Smith and I. Langmuir, Phys. Rev. 28, 727 (1926).

${ }^{18}$ I. B. Bernstein and I. Rabinowitz, Phys. Fluids 2, 112 (1959).

${ }^{19}$ J. D. Swift and M. J. R. Schwar, Electrical Probes for Plasma Diagnostics (Elsevier, New York, 1969).

${ }^{20}$ S. Humphries, Field Solutions on Computers (CRC, Boca Raton, 1998).

${ }^{21}$ W. R. Smythe, Static and Dynamic Electricity (McGraw-Hill, New York, 1968), 3rd ed., Chap. 1, p. 24.

${ }^{22}$ H. B. Dwight, Tables of Integrals and other Mathematical Data, 4th ed. (MacMillan, New York, 1961), pp. 179-186. 